a carcinogen and endocrine disruptor, causing the endometrial hyperplasia and EC in animal studies. Sweetened beverages cause a rise in insulin, which in turn inhibits sex-hormone binding protein. This results in higher levels of circulating free oestrogens. Also, insulin has mitogenic and anti-apoptotic properties, further inducing the endometrium proliferation. The favorable influence of regular physical activity on EC relapse and death is in accordance with previous studies, including recent meta-analysis.

Therefore, we encourage women treated for EC to consider reducing sweetened beverages and fried potatoes consumption and increasing physical activity.

Disclosures The authors have nothing to disclose.

\section{USE OF PREOPERATIVE AND INTRAOPERATIVE PARAMETERS FOR DECISION MAKING IN OVARIAN PRESERVATION IN ENDOMETRIAL ADENOCARCINOMA}

Halise Meltem Batur, Murat Gultekin, Mehmet Coskun Salman, Nejat Ozgul. Hacettepe University Faculty of Medicine; Department of Obstetrics and Gynaecology

\subsection{6/ijgc-2020-ESG0.62}

Introduction/Background Oophorectomy which is the integral part of surgery in endometrial adenocarcinoma leads to some adverse effects in premenopausal patients. Therefore, ovarian preservation concept has recently emerged especially in early stage disease. Several studies have shown that such approach does not adversely impact oncologic prognosis. This study aimed to retrospectively investigate the characteristics of endometrial adenocarcinoma patients with ovarian metastasis and to define criteria for ovarian preservation by using preoperative and intraoperative parameters.

Methodology Patients with endometrial adenocarcinoma who were operated at Hacettepe University Faculty of Medicine, Department of Obstetrics and Gynaecology were identified. The clinical and pathological characteristics of these patients were reviewed. Following univariate and multivariate analysis to determine factors associated with ovarian spread, different sets of criteria were analyzed to determine the subgroup of patients with no or negligible risk of ovarian metastasis.

Results The study group consisted of 725 patients and ovarian metastasis was detected in only $66(9.1 \%)$ of the patients. Univariate analysis showed tumor diameter, grade, histological type, myometrial invasion, peritoneal cytology, lymphovascular space invasion (LVSI), cervical invasion, omental and lymph node metastasis are significantly associated with ovarian metastasis while only LVSI, cervical invasion, omental and lymphatic involvement were significant on multivariate analysis. By using preoperative and intraoperative parameters only, no risk of ovarian metastasis was seen in patients of all ages with endometrioid tumor of any grade without myometrial invasion and risk was negligible $(0.7 \%)$ among 142 patients $(19.6 \%$ of study population) of any age with grade 1, endometrioid type tumor without deep myometrial invasion.

Conclusion Oophorectomy is not always necessary in endometrial adenocarcinoma. Preoperative and intraoperative uterusrelated factors may be used to define patients in whom ovarian preservation is safe similar to the approach used to determine surgical extent. Thus, ovaries may safely be preserved in almost $20 \%$ of patients with endometrial adenocarcinoma.

Disclosures No potential conflict of interest to declare.

\section{REAL-WORLD TREATMENT PATTERNS, HEALTHCARE RESOURCE USE, AND COSTS BY LINE OF THERAPY AMONG NEWLY DIAGNOSED ENDOMETRIAL CANCER PATIENTS}

${ }^{1}$ Chizoba Nwankwo, ${ }^{2}$ Ruchitbhai Shah, ${ }^{2}$ Nehemiah Kebede, ${ }^{2}$ Anuj Shah, ${ }^{2}$ Shelby Corman. ${ }^{1}$ Merck and Co., Inc; ${ }^{2}$ Pharmerit - an Open Health Company; Pharmerit International, Bethesda, MD, USA

\subsection{6/ijgc-2020-ESG0.63}

Introduction/Background Guidelines recommend surgery as primary therapy for endometrial cancer patients. Subsequent treatments can include radiation with/without systemic therapy depending on patients' prognosis. However, there is little data describing real-world treatment patterns and economic burden among newly diagnosed endometrial cancer patients. Therefore, this study aimed to assess real-world treatment patterns and healthcare costs by line of therapy (LOT) among newly diagnosed endometrial cancer patients.

Methodology Endometrial cancer patients newly diagnosed between January 2015 - June 2018 with continuous medical enrollment for 12 months prior and 6 months post diagnosis were identified in the Optum Clinformatics DataMart database. Treatments associated with endometrial cancer, including surgeries (bilateral salpingo-oophorectomy, hysterectomy and

Abstract 291 Table 1 Treatment patterns among newly diagnosed endometrial cancer patients by line of therapy

\begin{tabular}{|c|c|c|}
\hline Treatments & $\begin{array}{l}\text { First Line of } \\
\text { Therapy }\end{array}$ & $\begin{array}{l}\text { Second Line of } \\
\text { Therapy }\end{array}$ \\
\hline Surgery ${ }^{a}$ & $\mathrm{~N}=3,309$ & $\mathrm{~N}=47$ \\
\hline Hysterectomy & 3,274 (98.94\%) & $41(87.23 \%)$ \\
\hline Lymphadenectomy & $1,580(47.75 \%)$ & $20(42.55 \%)$ \\
\hline Bilateral salpingo-oophorectomy & $830(25.08 \%)$ & $11(23.40 \%)$ \\
\hline Radiation $^{2}$ & $\mathrm{~N}=884$ & $\mathrm{~N}=501$ \\
\hline Radiation only & $55(6.22 \%)$ & $330(65.87 \%)$ \\
\hline Radiation + systemic & $58(6.56 \%)$ & $163(32.53 \%)$ \\
\hline Radiation + surgery & $604(68.33 \%)$ & $4(0.8 \%)$ \\
\hline Radiation + surgery + systemic & $167(18.89 \%)$ & $4(0.8 \%)$ \\
\hline Systemic only a & $\mathrm{N}=152$ & $\mathrm{~N}=231$ \\
\hline Carboplatin & $49(32.24 \%)$ & $116(50.22 \%)$ \\
\hline Paclitaxel & $42(27.63 \%)$ & $100(43.29 \%)$ \\
\hline Megestrol acetate & $35(23.03 \%)$ & $12(5.19 \%)$ \\
\hline Other & $88(57.89 \%)$ & $144(62.34 \%)$ \\
\hline Systemic with surgery/radiation ${ }^{\text {a }}$ & $\mathrm{N}=780$ & $\mathrm{~N}=184$ \\
\hline Carboplatin & $529(67.82 \%)$ & $80(43.48 \%)$ \\
\hline Paclitaxel & $507(65.0 \%)$ & $74(40.22 \%)$ \\
\hline Cisplatin & $55(7.05 \%)$ & $12(6.52 \%)$ \\
\hline Other & $320(41.0 \%)$ & $122(66.3 \%)$ \\
\hline
\end{tabular}

a The categories are not mutually exclusive as patients may have received a combination of these therapies. Radiation therapy includes external beam radiotherapy and brachytherapy.

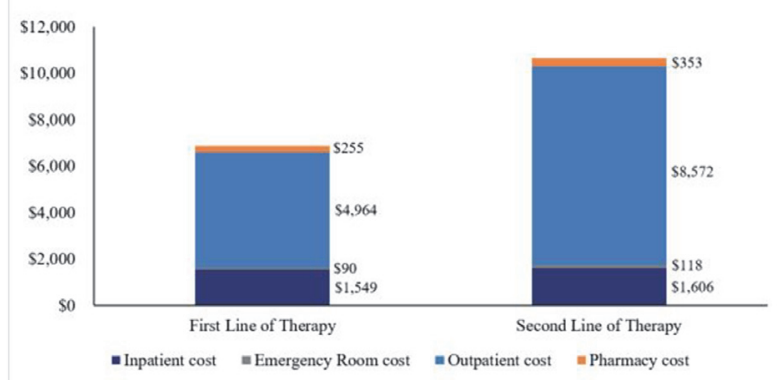

Abstract 291 Figure 1 Mean per patient per month healthcare costs by line of therapy among newly diagnosed endometrial cancer patients 
lymphadenectomy), radiotherapy (external beam radiotherapy and brachytherapy) and systemic therapies (chemotherapies, immunotherapies and hormonal therapies) were identified and described by LOT. The first treatment received post diagnosis was classified as LOT1. Treatments initiated within \pm 90 days of surgical procedures, 30 days of the end of a radiotherapy, and 28 days of the start of a systemic therapy were considered to be a part of the same LOT. Study outcomes included time to treatment initiation, most frequently received treatments in LOT1 and LOT2, and per patient per month (PPPM) costs attributable to LOT1 and LOT2.

Results Among 5,006 newly diagnosed endometrial cancer patients, 3,574 (71\%) received at least LOT1 and 771 $(15.4 \%)$ received LOT2. The median time from diagnosis to LOT1 initiation was $1.0(1.0-2.0)$ month. Hysterectomy (98.9\%) was the most common treatment in LOT1. Majority of patients received radiation therapy (65\%) in LOT2. Treatments received in LOT1 and LOT2 are summarized in table 1. The mean total healthcare cost from diagnosis to end of follow-up was \$6,088 PPPM. The PPPM costs attributable to each LOT are presented in figure 1 . The total healthcare costs during LOT2 exceeded those incurred during LOT1 with outpatient costs being the biggest driver.

Conclusion Newly diagnosed endometrial cancer patients received treatments consistent with guidelines with hysterectomy being the most common LOT1 treatment. Outpatient costs accounted for $70 \%-80 \%$ of total healthcare costs attributable to LOT1 and LOT2. Moving from LOT1 to subsequent LOTs was associated with an increase in healthcare costs which may be indicative of disease progression/ recurrence.

Disclosures This study was funded by Merck Sharp \& Dohme Corp., a subsidiary of Merck \& Co., Inc., Kenilworth, NJ, USA. Chizoba Nwankwo is an employee of Merck Sharp \& Dohme Corp., a subsidiary of Merck \& Co., Inc. Anuj Shah, Ruchit Shah, Shelby Corman, and Nehemiah Kebede are employees of Pharmerit, which received consulting fees related to this study.

\section{REAL-WORLD TREATMENT PATTERNS, HEALTHCARE RESOURCE USE, AND COSTS BY LINE OF THERAPY AMONG ENDOMETRIAL CANCER PATIENTS NEWLY INITIATING SYSTEMIC THERAPIES}

${ }^{1}$ Chizoba Nwankwo, ${ }^{2}$ Ruchitbhai Shah, ${ }^{2}$ Nehemiah Kebede, ${ }^{2}$ Anuj Shah, ${ }^{2}$ Shelby Corman. ${ }^{1}$ Merck and Co., Inc; ${ }^{2}$ Pharmerit - an Open Health Company; Pharmerit International, Bethesda, MD, USA

\subsection{6/ijgc-2020-ESGO.64}

Introduction/Background NCCN guidelines recommend the use of systemic therapy for women with advanced endometrial cancer. However, there are no data examining real-world treatment patterns and economic burden in this population. Therefore, this analysis described treatment patterns, and costs in a real-world cohort of endometrial cancer patients initiating systemic treatment.

Methodology Endometrial cancer patients with $\geq 2$ claims for a systemic therapy (i.e., chemo-, immuno- or hormonal therapies) within a 4-week period or a claim for an intrauterine device between June 2014 - September 2018 and having continuous medical enrollment for 6 months prior and 3 months post therapy initiation were identified in the Optum
Abstract 292 Table 1 Treatment patterns among endometrial cancer patients initiating systemic therapy by line of therapy

\begin{tabular}{|c|c|c|}
\hline Treatments & First Line of Therapy & Second Line of Therapy \\
\hline \multicolumn{3}{|c|}{ No prior presence of non-endometrial cancer } \\
\hline Systemic therapy only a & $\mathrm{N}=278$ & $\mathrm{~N}=80$ \\
\hline Carboplatin & $66(23.74 \%)$ & $11(13.75 \%)$ \\
\hline Megestrol acetate & $62(22.3 \%)$ & $14(17.5 \%)$ \\
\hline Paclitaxel & $58(20.86 \%)$ & $10(12.5 \%)$ \\
\hline Other & $176(63.31 \%)$ & $64(80 \%)$ \\
\hline Systemic therapy + radiation " & $\mathrm{N}=19$ & $\mathrm{~N}=9$ \\
\hline Carboplatin & $8(42.11 \%)$ & $4(44.44 \%)$ \\
\hline Megestrol acetate & $4(21.05 \%)$ & $2(22.22 \%)$ \\
\hline Paclitaxel & $7(36.84 \%)$ & $3(33.33 \%)$ \\
\hline Other & $10(52.63 \%)$ & $6(66.67 \%)$ \\
\hline \multicolumn{3}{|c|}{ Prior presence of non-endometrial cancer } \\
\hline Systemic therapy only a & $\mathrm{N}=1,933$ & $\mathrm{~N}=721$ \\
\hline Carboplatin & $791(40.92 \%)$ & $171(23.72 \%)$ \\
\hline Paclitaxel & $702(36.32 \%)$ & $147(20.39 \%)$ \\
\hline Megestrol acetate & $167(8.64 \%)$ & $66(9.15 \%)$ \\
\hline Other & $1251(64.72 \%)$ & $597(82.8 \%)$ \\
\hline Systemic therapy + radiation ${ }^{a}$ & $N=429$ & $\mathrm{~N}=67$ \\
\hline Cisplatin & $133(31 \%)$ & $9(13.43 \%)$ \\
\hline Carboplatin & $113(26.34 \%)$ & $13(19.4 \%)$ \\
\hline Paclitaxel & $95(22.14 \%)$ & $11(16.42 \%)$ \\
\hline Other & $251(58.51 \%)$ & $58(86.57 \%)$ \\
\hline
\end{tabular}

treatments listed under each category. Radiation therapy includes EBRT and brachytherapy.

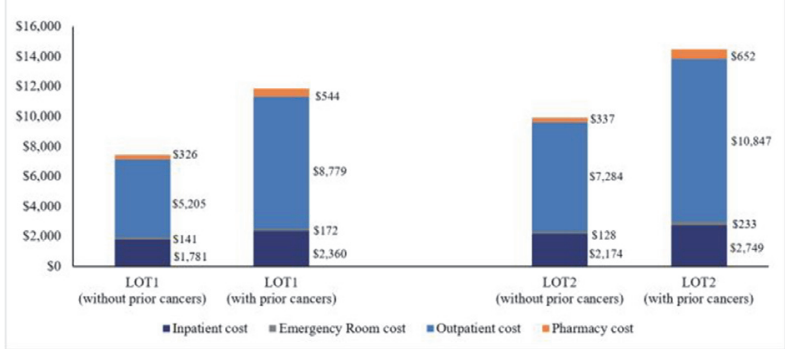

Abstract 292 Figure 1 Mean per patient per month healthcare costs by line of therapy among endometrial cancer patients newly initiating systemic therapy

Clinformatics DataMart database. Patients with endometrial cancer-related surgery performed within \pm 90 days of systemic therapy initiation were not included to exclude adjuvant use. All claims for the same systemic therapy without a $>90$-day gap or a new systemic treatment initiated within 28 days were a part of the same line of therapy (LOT). We reported the most frequently used treatments and per patient per month (PPPM) healthcare costs for LOT1 and LOT2. All analyses were stratified by the presence of non-endometrial cancers prior to systemic therapy initiation.

Results 2,659 women with endometrial cancer newly initiated systemic therapy (i.e., LOT1), 877 (32.98\%) received a LOT2, and $350(13.16 \%)$ had a LOT3. Most patients had a nonendometrial cancer (88.9\%) prior to initiating systemic therapy. The treatments received and associated costs in LOT1 and LOT2 are described in table 1 and figure 1, respectively. The median durations of LOT1 and LOT2 were 3.5 and 3.1 months, respectively. The proportions of patients receiving monotherapy in LOT1 and LOT2 were $55.3 \%$ and $54.4 \%$, respectively. The mean PPPM total healthcare expenditure over the entire follow-up was $\$ 11,109$ and outpatient costs $(\$ 8,073)$ accounted for $\sim 75 \%$ of this burden. Healthcare expenditure increased as patients moved from LOT1 to LOT2.

Conclusion Both taxanes and platinum-based therapies were used as the primary systemic treatments in this population. 